\title{
INDICACIONES Y UTILIDAD DE LA INMUNOGLOBULINA POR VÍA INTRAVENOSA EN EL MANEJO DE POLIMIOSITIS REFRACTARIA. REPORTE DE CASO Y REVISIÓN DE LA LITERATURA
}

\author{
Wilson A. Bautista M. MD. ${ }^{1 *}$, Elsa Retes S. MD. ${ }^{3}$, Claudia Mora MD. ${ }^{3}$, Claudia M. Guzmán V. MD. ${ }^{1}$, \\ John Londoño P. MD. ${ }^{3}$, Paola Varela MD. ${ }^{2}$, Rosa Salazar MD. ${ }^{2}$ Y Rafael R. Valle O. MD. ${ }^{3}$ \\ ${ }^{1}$ Residente del Programa de Reumatología, Universidad Militar Nueva Granada, Bogotá; ${ }^{2}$ Residente del Pro- \\ grama de Reumatología, Universidad de la Sabana, Bogotá; ${ }^{3}$ Reumatólogo, Servicio de Reumatología Hospital \\ Militar Central, Bogotá, Colombia, Universidad Militar Nueva Granada y Universidad de la Sabana
}

\begin{abstract}
Resumen
Las miopatías inflamatorias corresponden a un grupo heterogéneo de enfermedades adquiridas que comprometen el sistema muscular. Tienen en común la presencia de debilidad como síntoma predominante asociado a inflamación muscular. La polimiositis (PM) es una entidad subaguda que afecta principalmente adultos y compromete estructuras musculares proximales. En este artículo se presenta el caso de un paciente de 19 años de edad con diagnóstico confirmado de PM por clínica (compromiso muscular proximal, disfagia), por los resultados de las pruebas bioquímicas (elevación sérica de la creatina kinasa o $\mathrm{CK}$ ), por estudio de la ultraestructura por microscopia óptica de alta resolución y por los resultados de la resonancia magnética nuclear (RMN) de músculo, quien fue refractario al manejo inicial con corticoesteroides, azatioprina y ciclofosfamida. Con la administración de inmunoglobulina por vía intravenosa se observa una respuesta clínica favorable con aumento progresivo de la fuerza muscular. A pesar de que los esteroides constituyen el tratamiento de elección, en los casos refractarios se usan los agentes inmunosupresores, y la recomendación para su utilización depende de la experiencia médica o conocimiento empírico y de los resultados de los estudios de análisis de seguridad y eficacia. La inmunoglobulina por vía IV es ampliamente usada en el tratamiento de varias enfermedades autoinmunes y su eficacia radica en el efecto inmunomodulador. Sin embargo, es necesario realizar estudios clínicos controlados adicionales, que evalúen su eficacia y seguridad en pacientes con PM.
\end{abstract}

Palabras clave: miopatía, polimiositis, esteroides

\section{INDICATIONS AND UTILITY OF INTRAVENOUS IMMUNOGLOBULIN IN THE TREATMENT OF REFRACTORY POLYMYOSITIS. A CASE REPORT AND LITERATURE REVIEW}

\begin{abstract}
Inflammatory myopathies belong to a heterogeneous group of acquired diseases involving the muscular system. The predominant symptom is weakness associated with muscle soreness. Polymyositis $(\mathrm{PM})$ is a disorder that primarily affects adults and involves proximal muscle structures. Here, a case report of a 19-year-old patient diagnosed with PM is presented as confirmed by clinical evidence (involving proximal muscle, dysphagia), biochemical findings (elevated serum CK), high-resolution optical microscopy, and ultra structure and nuclear magnetic resonance of muscle. The patient was
\end{abstract}

* Correspondencia: Wilson A. Bautista M. wbatu@hotmail.com Dirección postal: Servicio de Reumatología, Hospital Militar Central Transversal 3 No. 49-00, Bogotá, Colombia.

Recibido: Octubre 27 de 2010 Aprobado: Diciembre 15 de 2010 
refractory to initial treatment with corticosteroids, azathioprine and cyclophosphamide. With the administration of intravenous immunoglobulin a good clinical response was observed with progressive increase in muscle strength. Although steroids constitute the main treatment, in refractory cases the use of immunosuppressive agents is recommended, base on medical experience or empirical knowledge together with the analysis of safety and efficacy. Intravenous immunoglobulin is widely used in the treatment of various autoimmune diseases and its effectiveness relies on immunomodulatory effect. Controlled clinical studies are needed to evaluate further its efficacy and safety in patients with PM.

Key words: miopathies, polymyositis, steroids

\title{
INDICAÇÕES E UTILIDADE DA IMUNOGLOBULINA INTRAVENOSA NA ABORDAGEM DE POLIMIOSITE REFRATÁRIO. RELATO DE CASO E REVISÃO DA LITERATURA
}

\section{Resumo}

\begin{abstract}
As miopatias inflamatórias pertencem a um grupo heterogêneo de doenças adquiridas, envolvendo o sistema muscular. Têm em comum a presença de fraqueza como sintoma predominante associada a inflamação musculares. A polimiosite (PM) é uma entidade subaguda que afeta principalmente adultos e envolve estruturas musculares proximais. Este artigo apresenta o caso de um doente de 19 anos com diagnóstico confirmado de PM por clínica (envolvendo a musculatura proximal, disfagia), pelos resultados de testes bioquímicos (níveis séricos elevados de creatinoquinase ou $\mathrm{CK}$ ), para o estudo da ultra-estrutura por microscopia óptica de alta resolução y por los resultados de la resonancia magnética nuclear (RMN) de músculo, que era refratária ao tratamento inicial com corticóides, azatioprina e ciclofosfamida. Com a administração de imunoglobulina intravenosa há uma resposta clínica favorável com aumento progressivo da força muscular. Embora os esteróides são o tratamento de escolha, em casos refratários, são utilizados os imunossupressores, e a recomendação para o uso depende da experiência médica ou conhecimento empírico e os resultados dos estudos de análise de segurança e eficácia. A imunoglobulina intravenosa é amplamente utilizado no tratamento de várias doenças auto-imunes e sua eficácia está no efeito imunomodulador. No entanto, é necessário realizar mais estudos clínicos controlados que avaliem sua eficácia e segurança em pacientes com PM.
\end{abstract}

Palabras-chave: miopatias, polimiositis, esteróides

\section{Introducción}

Las miopatías inflamatorias corresponden a un grupo heterogéneo de enfermedades adquiridas agudas, subagudas y crónicas que comprometen el sistema muscular, poco frecuentes, con una incidencia anual de 5 a 10 casos por millón y una prevalencia de 50 90 casos por millón. Presenta una incidencia bimodal con un pico en la infancia (5 - 15 años) y otro en la vida adulta (30 - 50 años) (6). Presentan como síntoma común la debilidad muscular que varía de intensidad, de moderada a severa, asociado a la inflamación muscular (1-3). Son desórdenes clínicamente importantes debido a que pueden presentar tasas de morbilidad y mortalidad considerables si no se realiza un diagnóstico temprano, y como consecuencia hay una progresión de la debilidad muscular que amenaza la vida y complicaciones cardiacas y pulmonares $(4,5)$. Esta entidad fue descrita en 1880 por médicos alemanes, pero el término de PM fue introducido por E. Wagner en 1886 y el de dermatomiositis (DM) por H. Unverricht (7).

Actualmente se dispone de la RMN, un procedimiento de estudio no invasivo y con aplicación cada vez más creciente especialmente para el diagnóstico de enfermedades del sistema músculo- esquelético. En los procesos inflamatorios se altera la distribución del agua en los tejidos, lo que condiciona diferentes tiempos de expansión y como consecuencia en la resonancia, se observan cambios en la intensidad o contraste de la 
señal, registrándose mayor brillantez en las imágenes proyectadas de los músculos (8). En cuanto al tratamiento, los esteroides constituyen el pilar del manejo de este tipo de pacientes, y la elección de otras opciones terapéuticas tales como agentes inmunosupresores en los casos refractarios, se hace de manera empírica y depende de la experiencia médica y de los estudios de eficacia y de seguridad. Desafortunadamente se han realizado pocos ensayos clínicos controlados, por lo que sería interesante diseñar estudios que incluyan pacientes con $\mathrm{DM}$ y miositis de cuerpos de inclusión, que permitan conocer esquemas terapéuticos adicionales que sean validados por datos experimentales.

\section{Reporte de caso}

Se presenta el caso de un paciente, soldado profesional de 19 años de edad, procedente de San José del Guaviare, Colombia. Ingresa remitido a la institución con cuadro clínico de dos meses de evolución consistente en pérdida progresiva de la fuerza muscular en miembros inferiores, de predominio proximal, asociado a parestesias y dolor de leve intensidad. Dos semanas después presenta compromiso adicional de miembros superiores, trastorno de deglución para sólidos y líquidos, limitación para movimientos oro faciales, articulación de palabras, dificultad para sedestación prolongada e incapacidad para mantener el tono postural.

Todos sus antecedentes médicos, quirúrgicos, toxicológicos y de alergias eran negativos. Al examen físico presentaba compromiso de fuerza muscular $2 / 5$ en miembros superiores y $3 / 5$ en miembros inferiores, xerodermia generalizada y no se observaron lesiones en piel. Durante la hospitalización se ordenaron los siguientes paraclínicos y sus resultados fueron: hemograma y función renal sin alteraciones, transaminasas tales como la enzima aspartato aminotransferasa (AST): $637 \mathrm{U} / \mathrm{L}$ y la alanino aminotransferasa (ALT): $358 \mathrm{U} / \mathrm{L}$, la CK al ingreso: 13.832 con fracción MB: 1905. Se le realizó electromiografía y velocidades de neuroconducción. Los resultados eran compatible con un proceso miopático activo de predominio en miembros superiores. Otros resultados: TSH: 11,8 Unidades/ ml. ANAS: 1/1280 (Técnica Inmunofluorescencia Indirecta con células Hep-2) con patrón moteado grueso.

También se le realizaron RMN de cintura escapular que mostró alteraciones difusas de la intensidad de la señal, con aumento de la intensidad en los grupos musculares observados (figura 1) y biopsia muscular de cuádriceps en la que se reporta infiltrados inflamatorios multifocales de predominio linfocítico que rodea e invade fibras musculares sanas. El estudio de la ultraestructura por microscopia óptica de alta resolución mostró cambios miopáticos con fibras de marcada irregularidad en el tamaño y colágeno endomisial aumentado en forma severa y se observaron fibras atróficas con sarcómeras desordenadas sin claro reconocimiento de las fibras ni de las bandas. No se observaron inclusiones sarcoméricas o nucleares de ningún tipo con heterocromatina normal.

Basados en el cuadro clínico, los resultados de los paraclínicos y el diagnóstico confirmado, se inició manejo con prednisona inicialmente a $1 \mathrm{mg} / \mathrm{kg} /$ día y posteriormente a $2 \mathrm{mg} / \mathrm{kg} /$ día por falta de respuesta clínica. Posteriormente se decide adicionar azatioprina como agente inmunomodulador y ahorrador de esteroides con aumento progresivo de la dosis hasta $150 \mathrm{mg} /$ día con persistencia de CKs elevadas (mayor a $4000 \mathrm{U}$ ) y trastorno de deglución persistente.

El paciente permanecía estacionario en su evolución clínica y en ocasiones con tendencia al deterioro de la fuerza muscular a pesar del tratamiento instaurado, por lo cual se decide en Junta de Decisiones del Servicio, el inicio de tratamiento con bolos de ciclofosfamida $600 \mathrm{mg} / \mathrm{m}^{2}$ de superficie corporal, recibió dos dosis sin evidencia de mejoría clínica. Posteriormente se decide administración de $2 \mathrm{~g} / \mathrm{kg}$ de inmunoglobulina por vía endovenosa, con lo cual tres días después de su administración, inicia deambulacion asistida, deglución de alimentos semisólidos, aumento de movimientos orofaciales y sedestación prolongada, por lo cual se continuó de forma programada con bolos cada seis semanas.

\section{Polimiositis como entidad clínica}

Se considera la PM como una miopatía subaguda que se manifiesta en el curso de semanas a meses, afecta principalmente adultos, raramente niños y compromete estructuras musculares proximales. A diferencia de la DM su inicio no suele ser claramente identificado (9). Como única entidad es poco común y con frecuencia mal diagnosticada, y se recomienda hacer el diagnóstico diferencial especialmente con la miositis por cuerpos de inclusión. Sin embargo, es necesario descartar otras patologías tales como distrofias musculares y miopatías endocrinas o tóxicas (Tabla 1) $(10,11)$. 


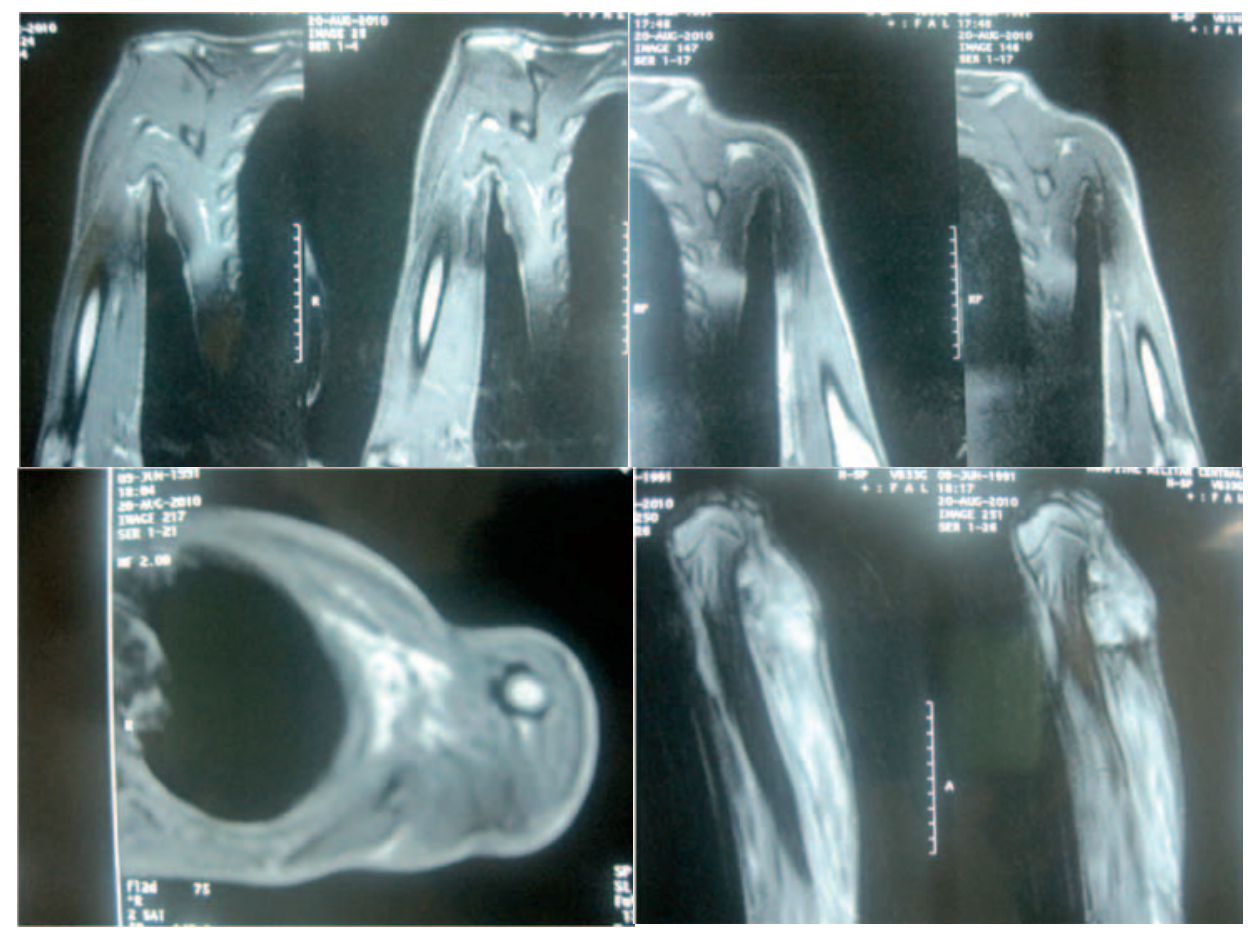

Figura 1. RMN de cintura escapular. Aumento de la intensidad de la señal de los grupos musculares proximales del miembro superior. Dicha alteración de la señal es homogénea y con un grado de compromiso diferente.

Tabla 1. Diagnóstico diferencial del complejo dermatomiositis - polimiositis.

\section{Miopatías Inflamatorias}

\section{Polimiositis}

Dermatomiositis

Miositis asociada a otras enfermedades del colágeno y vasculitis Miositis asociada a neoplasia maligna

\section{Otras formas de miopatía inflamatoria}

Miositis asociada a eosinofilia Miositis localizada o focal

\section{Miopatía causada por Infección}

Adenovirus, citomegalovirus, coxsakie, hepatitis B, virus de inmunodeficiencia Humana, cándida, criptococo, estafilococo, estreptococo, tuberculosis, otros.

\section{Miopatía causada por fármacos y toxinas}

Cloroquina, colchicina, D-penicilamina, glucocorticoides, etc.

Existen muchos criterios para la clasificación (Tabla 2) y varias clasificaciones de esta enfermedad, pero la que más se utiliza en la práctica clínica es la de Bohan y Peter (Tabla 3) $(6,12,13)$. 
Tabla 2. Criterios Diagnósticos para Dermatomiositis, Polimiositis.

\begin{tabular}{|c|c|}
\hline CRITERIO & DEFINICIÓN \\
\hline Debilidad muscular proximal & $\begin{array}{l}\text { Debilidad muscular en la } \\
\text { cintura escapular y pélvica }\end{array}$ \\
\hline $\begin{array}{l}\text { Enzimas sarcoplásmicas } \\
\text { elevadas }\end{array}$ & $\begin{array}{l}\text { Elevación de enzimas muscu- } \\
\text { lares en suero }\end{array}$ \\
\hline $\begin{array}{l}\text { Cambios miopáticos en la } \\
\text { Electromiografía }\end{array}$ & $\begin{array}{l}\text { Potenciales de acción caracte- } \\
\text { rísticas de miopatía }\end{array}$ \\
\hline Biopsia muscular & $\begin{array}{l}\text { Inflamación crónica con } \\
\text { degeneración y regeneración } \\
\text { de fibras musculares. }\end{array}$ \\
\hline $\begin{array}{l}\text { Pápulas de Gottron o Helio- } \\
\text { tropo }\end{array}$ & $\begin{array}{l}\text { Pápulas planas en los nudillos } \\
\text { de los dedos. Rash hiperémico } \\
\text { alrededor de los párpados }\end{array}$ \\
\hline
\end{tabular}

La presencia de los primeros cuatro criterios hace el diagnóstico de PM. Si hay cambios características en la piel se realiza el diagnóstico de DM.

Tabla 3. Clasificación del complejo Dermatomiositis/Polimiositis propuesto por Bohan y Peter.

\begin{tabular}{ll}
\hline Grupo 1 & Polimiositis Idiopática Primaria \\
\hline Grupo 2 & Dermatomiositis Idiopática Primaria \\
\hline Grupo 3 & $\begin{array}{l}\text { Dermatomiositis (o Polimiositis) asociada a } \\
\text { neoplasia }\end{array}$ \\
\hline Grupo 4 & $\begin{array}{l}\text { Dermatomiositis (o Polimiositis) infantil asociada } \\
\text { a Vasculitis }\end{array}$ \\
\hline Grupo 5 & $\begin{array}{l}\text { Polimiositis o Dermatomiositis asociada con enfer- } \\
\text { medad del colágeno (Síndrome de Superposición) }\end{array}$ \\
\hline
\end{tabular}

\section{Polimiositis refractaria al manejo convencional}

Los objetivos del tratamiento son mejorar la habilidad para desarrollar actividades básicas cotidianas incrementando la fuerza muscular y controlando las manifestaciones extra musculares (disfagia, disnea, artralgias). Dentro de las miopatías las DM responden mejor al tratamiento convencional que las PM y las más difíciles de tratar son las miopatías por cuerpos de inclusión $(14,15)$.

\section{Corticoesteroides}

La prednisona es el primer medicamento de elección pero la decisión de utilizarse de forma continua, se basa en la experiencia y conocimiento empírico del médico. La administración de dosis de 80 a 100 mg/día durante tres o cuatro semanas permite, en la mayoría de casos, disminuir la dosis gradualmente hasta completar diez semanas de tratamiento. Aunque muchos pacientes responden en algún grado y por determinado tiempo, algunos se convierten en refractarios a los esteroides y por lo tanto requieren de la administración de agentes inmunosupresores. En algunos casos es necesario decidir iniciar la administración de agentes inmunosupresores, como por ejemplo: 1- si a pesar de que hay una buena respuesta clínica a los esteroides, no se deben utilizar para evitar efectos adversos (necrosis ósea avascular, osteoporosis); 2- si están utilizando dosis altas de esteroides (mayores de $2 \mathrm{mg} / \mathrm{kg}$ ) y no se pueden disminuir por temor a recaídas; 3 - si después de dos o tres meses de tratamiento con altas dosis de prednisona, el paciente no responde y muestra debilidad muscular progresiva y falla respiratoria $(14,15)$.

\section{Agentes inmunosupresores}

Como se ha mencionado ya, la decisión de elegir el uso de agentes inmunosupresores en los casos que son refractarios al tratamiento de primera elección, depende de la experiencia médica o el conocimiento empírico y de los resultados de los análisis de seguridad y de eficacia. Se ha demostrado que la utilización de 2,5-3,0 mg/kg de azatioprina en este tipo de pacientes ayuda a su recuperación (17). Su eficacia es soportada por un pequeño número de reporte de casos en miositis inflamatorias y en estudios retrospectivos se ha observado que cerca del $75 \%$ de pacientes tratados con este medicamento, muestran una buena respuesta $(18,19)$. En el caso de metotrexate, hay pocos reportes de casos y estudios retrospectivos que demuestren su eficacia en adolescentes con miositis inflamatorias idiopáticas (20). Se ha observado que la utilización de la ciclosporina en dosis de $3-10 \mathrm{mg} / \mathrm{kg} /$ día fue efectiva en pacientes con miositis inflamatoria y en quienes la combinación de esteroides y otros inmunosupresores había fallado (21). Aunque se han publicado varios reportes favorables de la utilización de la ciclofosfamida, este ha sido controversial y su indicación está restringida a pacientes que no responden a corticoesteroides o a otra combinaciones de fármacos (22).

\section{Inmunoglobulina por vía IV}

Es ampliamente usada en el tratamiento de varias enfermedades autoinmunes y su eficacia radica en su efecto inmunomodulador. Gelfang (23) sugirió que puede ser usada para el tratamiento de miositis inflamatoria idiopática en dosis de $1 \mathrm{~g} / \mathrm{kg}$ día por dos días cada cuatro semanas, y los parámetros que utilizaron 
para medir su eficacia fueron el incremento en la fuerza muscular y la disminución de CPK. Dalakas (14) realizó un ensayo aleatorizado, doble ciego, controlado con placebo en quince pacientes con dermatomiositis refractaria y los resultados obtenidos mostraron que los pacientes presentaron un aumento del índice de fuerza muscular estadísticamente significativo, y en las biopsias se observaba una mejoría de los hallazgos histopatológicos (24).

Aunque en pacientes con PM no se han hecho estudios controlados, se ha observado que el uso de la inmunoglobulina de aplicación IV es efectiva en el $70 \%$ de los pacientes, de donde se concluye que es una opción de tratamiento viable en pacientes con PM; sin embargo, se requiere diseñar otros estudios que valoren su seguridad y eficacia en pacientes con miositis inflamatoria idiopática. También se ha observado que es útil en pacientes con disfagia $(31,32)$.

\section{Utilidad de la RMN musculoesquelética en el diagnóstico de las miopatías}

El diagnóstico de las miopatías inflamatorias se basa usualmente en los criterios clínicos establecidos, en los resultados de los exámenes paraclínicos y en el resultado de la biopsia muscular en los casos que se requiera (25). Dentro de un grupo muscular determinado, la distribución del proceso inflamatorio pude ser heterogénea, pero en el caso de las miopatías inflamatorias idiopáticas pueden estar confinada a un solo músculo; por lo tanto, para evitar una interpretación errada cuando se observan las imágenes de músculo debido a la toma inadecuada de las muestras, es muy útil la identificación del sitio de la toma de la biopsia $(26,27,28)$.

En RMN de músculos, es importante seleccionar las secuencias adecuadas para evitar las interferencias en la interpretación de las imágenes; dado que la grasa puede ocasionar este tipo de inconveniente, se utilizan las secuencias T2 o la STIR (short tau inversión recovery) para eliminar las señales que generan. Cuando se utiliza la secuencia T2, los músculos con edema inflamatorio se observan hiperintensos (blancos), mientras que aquellos menos comprometidos emiten una baja señal (30). La importancia de la RMN se debe a que ayuda a disminuir el número de resultados falsos negativos, los cuales pueden presentarse en un 10 al $25 \%$ de los casos cuando no se utiliza ayuda de imágenes; de igual forma, es una herramienta que ayuda a identificar las áreas de actividad de la enfermedad, permitiendo así tomar las biopsias en los sitios apropiados (28-30).

\section{Conclusiones}

La polimiositis es una entidad subaguda que afecta principalmente adultos y compromete estructuras musculares proximales; el tratamiento de elección son los esteroides y en los casos que son refractarios a este medicamento, se usan agentes inmunosupresores; la recomendación para su utilización depende de la experiencia médica o conocimiento empírico y de los resultados de los análisis de seguridad y de eficacia del mismo. La inmunoglobulina por vía intravenosa es ampliamente usada en el tratamiento de varias enfermedades autoinmunes por su efecto inmunomodulador. Aunque la inmunoglobulina aplicada IV parece ser efectiva en dichos pacientes, en polimiositis no se han realizado estudios controlados; por tal razón, se hace necesario diseñar estudios multicéntricos (dada la baja incidencia de la enfermedad en la población), que permitan conocer esquemas terapéuticos adicionales que sean validados por datos experimentales. La RMN es un procedimiento de estudio no invasivo, que es utilizado cada vez más, para el diagnóstico de enfermedades del sistema músculo-esquelético.

\section{Referencias}

1. Dalakas M C. Polymyositis, Dermatomyositis and Inclusion-body Myositis. N Engl J Med. 1991;325:1487-98.

2. Engel A G, Hohlfeld R, Banker B Q. The Polymyositis and Dermatomyositis Syndromes. In: Engel A G, Franzini-Armstrong C, eds. Myology, 2nd ed. New York: McGraw-Hill, 1994: p. 1335-83.

3. Mastaglia F L, Phillips B A. Idiopathic Inflammatory Myopathies: Epidemiology, Classification and Diagnostic Criteria. Rheum Dis Clin N Am. 2002;28:723-41.

4. Danko K, Constantin T, Borgukya G, Szegedi G. Long Term Survival of Patients with Idiopathic Inflammatory Myopathies According to Clinical Features: A Longitudinal Study of 162 Cases. Medicine. 2004;83:35-42.

5. Marie I, Hachulla E, Hatron P Y, Hellot M F, Levesque H, Devulder B, et al. Polymiositis and Dematomyositis: Short Term and Long Term Outcome, and Predictive Factors. J Rheumatol. 2001;28:2230-7.

6. Restrepo J F. Dermatomiositis, Polimiositis. Rev Col Reum. 2003;10(2):135-41.

7. Wagner E. Ein Fall Von Akuter Polymyositis. Deutsch Arch klin Med. 1886;40:241-66.

8. Fraser DD, Frank J, Dalakas M. Magnetic Resonance Imaging in the Idiopathic Inflammatory Myopathies. $J$ Rheumatol. 1991;18(11):1693-99.

9. Dalakas M C. Diseases of Muscle and the Neuromuscular Junction. Sci Am. 1997;11:1-14. 
10. Dalakas M C, Hohlfeld R. Seminar: Polymyositis and Dermatomyositis. Lancet. 2003;362:971-82

11. Guzman C, Cubides M F, Reyes E. Miopatia por Cuerpos de Inclusión. Caso Clínico y Revision de la Literatura. Revista Med. 2010;18(1):93-9.

12. Bohan A, Peter J. Polymyositis and Dermatomyositis (First of two parts). N Engl J Med. 1975;292(2):344-47.

13. Bohan A, Peter J. Polymyositis and Dermatomyositis (Second of two parts). N Engl J Med. 1975;292(8):403-07.

14. Dalakas M C. How to Diagnose and Treat the Inflammatory Myopathies. Semin Neurol. 1994;92:365-69.

15. Oddis C V. Idiopathic Inflammatory Myopathy: Management and Prognosis. Rheum Dis Clin N Am. 2002;28:979-1001.

16. Dalakas M C. Polymyositis, Dermatomyositis and Inclusion Body Myositis. In: Braunwald E, Fauci A S, Kasper D L, Hauser S L, Longo D L, Jameson J L, eds. Harrison's Principles of Internal Medicine, 15th ed. New York: McGraw-Hill, 2001; p. 2524-29.

17. Bunch T W, Worthington J W, Combs J J, Ilstrup D M, Engel A G. Azathioprine and Prednisone for Polymyositis: A Controlled Clinical Trial. Ann Intern Med. 1980;92:365-69.

18. Ramirez G, Asherson R A, Khamashta M A, Cervera R, D'Cruz D, Hughes G R. Adult-onset Polymyositis-Dermatomyositis: Description of 25 Patients with Emphasis on Treatment. Semin Arthritis Rheum. 1990;20:114-20.

19. Bunch T W. Prednisone and Azathioprine for Polymyositis: Long-term Followup. Arthritis Rheum. 1981;24:45-8.

20. Zieglschmid-Adams M E, Pandya A, Cohen S B, Sontheimer RD. The Value of Methotrexate in Dermato-Myositis. J Am Acad Dermatol. 1998;38:130-2.

21. Zeller V, Cohen P, Prieur A M, Guillevin L. Cyclosporin A Therapy in Refractory Juvenile Dermatomyositis. Experience and Longterm Followup of 6 cases. J Rheumatol. 1996;23:1424-7.
22. De Vita S, Fossaluzza V. Treatment of Idiopathic Inflammatory Myopathies with Cyclophosphamide Pulses: Clinical Experience and a Review of the Literature. Review. Acta Neurol Belg. 1992;92:215-27

23. Gelfand E W. The Use of Intravenous Immune Globulin in Collagen Vascular Disorders: A Potentially New Modality of Therapy. J Allergy Clin Immunol. 1989;84:613-5.

24. Dalakas M C, Illa I, Dambrosia J M. A Controlled Trial of Highdose Intravenous Immune Globulin Infusions as Treatment for Dermatomyositis. N Engl Med. 1993;329:1993-2000.

25. Dalakas M C, Hohlfeld R. Polymyositis and Dermatomyositis. Lancet. 2003;362:971-982.

26. Maillard SM, Jones R, Owens C. Quantitative Assessment of MRI T2 Relaxation Time of Thigh Muscles in Juvenile Dermatomyositis. Rheumatology. 2004;43:603-608.

27. Connor A, Stebbings S, Anne H N. STIR MRI to Direct Muscle Biopsy in Suspected Idiopathic Inflammatory Myopathy. J Clin Rheumatol. 2007;13:34-345.

28. O'Connell M J, Powell T, Brennan D. Whole-body M R Imaging in the Diagnosis of Polymyositis. A J R Am J Roentgenol. 2002;179:967-971.

29. Carrino J A, Blanco R. Magnetic Resonance: Guided Musculoskeletal Interventional Radiology. Semin Musculoskelet Radiol. 2006;10:159-74.

30. Ulrich A W. Imaging Tools for the Clinical Assessment of Idiopathic Inflammatory Miositis. Current Opinion in Rheumatology. 2008;20:656-61.

31. Dalakas M. Inmunotherapy of Miositis: Issues, Concerns and Future Prospects. Nat Rev Rheumatol. 2010;6:129-37.

32. Dalakas M C. Intravenous Immunoglobulin in Autoimmune Neuromuscular Diseases. JAMA. 2004;291:2367-75. 